\title{
Microscopy Techniques in Pharmaceutical and other Manufacturing Industries
}

\author{
Diane Alonso
}

Sanofi-Aventis, 10236 Marion Park Drive, Kansas City, MO 64134

Microscopy provides the manufacturer with a valuable tool for the identification of a wide variety of materials including fibers/polymers, metal particles, blemishes, wood particles and many other materials. This presentation will illustrate microscopy techniques and instrumentation used to isolate, identify and document particulates.

Setting up the microscopy laboratory involves acquiring the appropriate instrumentation and one or two key individuals that have the training and experience to operate the instrumentation and interpret resulting data. A well-equipped microscopy laboratory starts with a good polarized light microscope and imaging equipment. Other spectroscopy instrumentation would include a microscope hot-stage, fourier transform infrared microscope (FTIR), raman micro stage, scanning electron microscope (SEM), energy dispersive xray spectrometer (EDXS) and others.

Figure 1 illustrates a good example of how microscopy can be used to identify minor blemishes. Visual particulate from extraneous minerals can be difficult to source and isolate, but common raw materials such as talc can contain trace amounts of graphite and other minerals that cause spots on tablets, beads and in cosmetics. Graphite has no distinctive characteristics when analyzed on common spectroscopy equipment. The unique visual characteristics of graphite can be identified by the polarized light microscope [1].

Metal particles (wear products) are another common problem to industry. Figure 2 illustrates a tablet spot caused by a metal particle. Identification can be done using PLM and microchemical techniques [2]. Semi-quantitative information can be obtained by energy dispersive x-ray spectroscopy. Elemental data from the unknowns gives important alloy information and can than be compared to control materials. Equipment surfaces and machine parts are a routine source of particulate metal. Figure 2 shows an EDXS spectrum and elemental map of an iron and zinc metal particle that was found to be from a zinc plated iron drum ring closure.

Figure 3 is an example of the identification of a polymer. The isolate can be identified by morphology using the polarized light microscope. Further identification techniques include melting point and infrared spectroscopy. Figure 3 shows the infrared spectra of the unknown compared to a control sample, which identified it as hot melt glue [3].

\section{References}

[1] The Particle Atlas, 2nd ed., MicroDataware, 1992

[2] Chamot and Mason, Handbook of Chemical Microscopy, 2nd ed., republication by McCrone Research Institute in cooperation with John Wiley and Sons., Inc., 1989

[3] I would like to acknowledge the McCrone Research Institute (McRI), Chicago Illinois, and their certification program. 

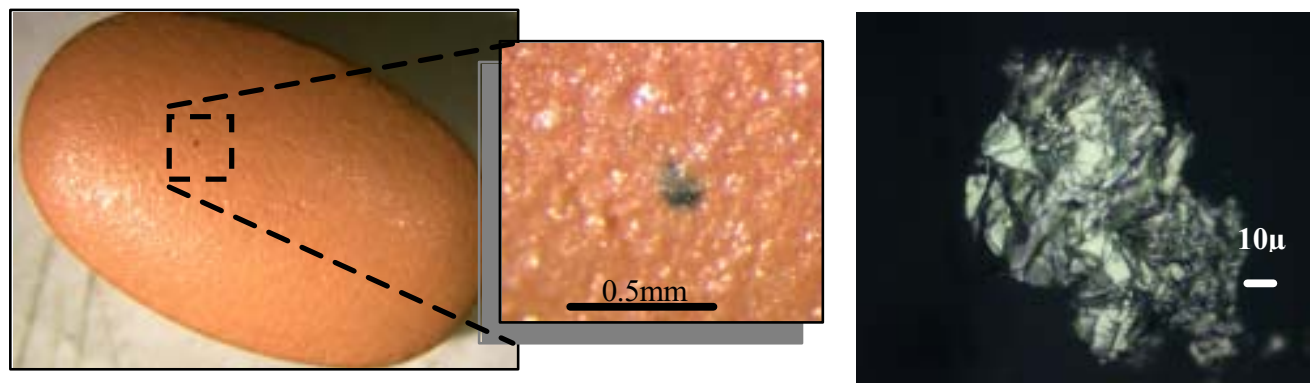

Figure 1. A spot on a tablet from graphite. Graphite particle viewed in reflected crossed polarized light illustrates metallic luster and flaky habit.
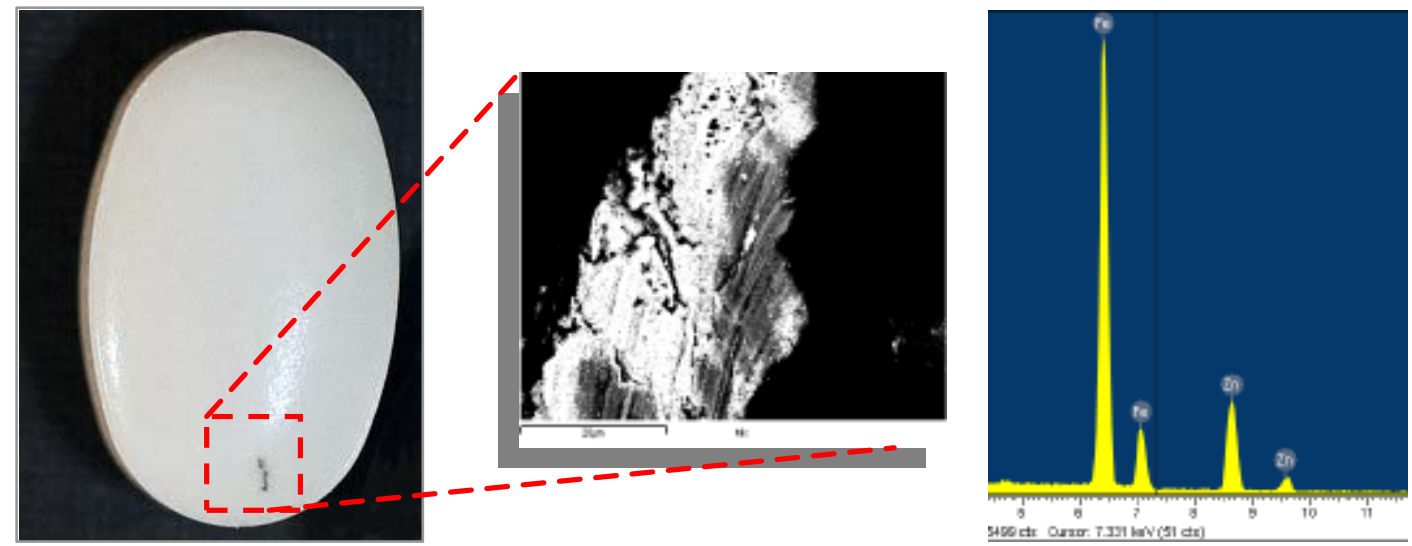

Figure 2. Metal particle in tablet subsequently isolated and imaged with SEM. EDXS spectrum of the metal particle also can produce an element map representing the areas filled by iron (light gray) and zinc (dark gray).
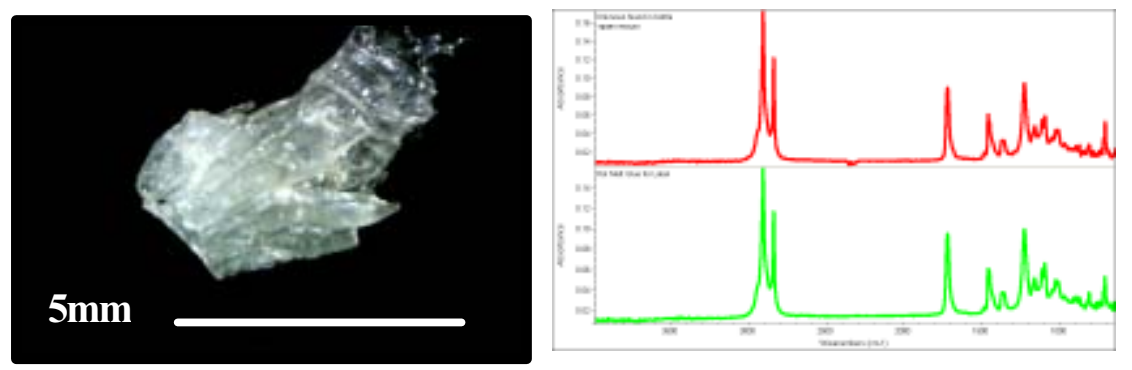

Figure 3. Unknown particle is identified as hot melt glue by FTIR. 